ISSN 0103-5150

Fisioter. Mov., Curitiba, v. 26, n. 3, p. 617-622, jul./set. 2013

Licenciado sob uma Licença Creative Commons

\title{
Efeitos das Correntes diadinâmicas de Bernard sobre a nociceptividade e edema no trauma tendíneo de ratos
}

\author{
Bernard's diadynamics effects on the nociception \\ and edema in rats tendon injury
}

\author{
Jhenifer Karvat ${ }^{[a]}$, Anamaria Meireles ${ }^{[b]}$, Bruno Pogorzelski Rocha ${ }^{[b]}$, Camila Thieimi Rosa ${ }^{[a]}$, \\ Lígia Inez Silva ${ }^{[b]}$, Gladson Ricardo Flor Bertolini ${ }^{[c]}$
}

[a] Acadêmicas do curso de Fisioterapia da Universidade Estadual do Oeste do Paraná (Unioeste), Cascavel, PR - Brasil, e-mails: jhennykarvat@hotmail.com,camilathieime@hotmail.com

[b] Fisioterapeutas graduados pela Universidade Estadual do Oeste do Paraná (Unioeste), Cascavel, PR - Brasil, e-mails: anammeireles@yahoo.com.br,brunop.rocha@yahoo.com.br, ligiainezsilva@hotmail.com

[c] Doutor em Ciências da Saúde Aplicadas ao Aparelho Locomotor pela Faculdade de Medicina de Ribeirão Preto da Universidade de São Paulo (FMRP/USP), docente do curso de Graduação em Fisioterapia e do Mestrado em Biociências e Saúde na Universidade Estadual do Oeste do Paraná (Unioeste), Cascavel, PR - Brasil, e-mail: gladsonricardo@gmail.com

\section{Resumo}

Introdução: O tratamento fisioterapêutico é utilizado para minimizar prejuízos do processo inflamatório. Destaca-se o uso da eletroterapia, sendo as Correntes diadinâmicas de Bernard (CDB) um dos recursos possíveis, porém com poucas evidências na literatura. Objetivo: Analisar os efeitos das CDB sobre a nociceptividade e edema no trauma tendíneo de ratos. Materiais e métodos: A amostra foi composta por 18 ratos Wistar, divididos em: grupo controle (GC); grupo do polo negativo (GP-); e grupo do polo positivo (GP+). Os animais tiveram seu tendão calcâneo direito, traumatizado com energia de 0,4 J. Para avaliar a nociceptividade, utilizou-se o analgesímetro digital tipo "Von Frey", o qual se aplicou no tendão lesado. E para avaliar o edema utilizou-se um paquímetro. 0 tratamento foi realizado com as correntes DF, CP e LP (3 minutos cada), diariamente, em cindo sessões de terapia. Resultados: Na avaliação da nociceptividade, houve diferença significativa com o momento pós-lesão, somente nas duas últimas avaliações no GP+. Na avaliação do edema obteve-se retorno aos valores basais apenas 
para GP+. Conclusão: Somente o polo positivo das diadinâmicas de Bernard foi eficaz em diminuir a nociceptividade e o edema em ratos submetidos a trauma no tendão de calcâneo.

Palavras-chave: Tendão do calcâneo. Estimulação elétrica nervosa transcutânea. Medição da dor.

\section{Abstract}

Introduction: Physical therapy is used to minimize inflammatory process damage, and highlight the use of electrotherapy. Bernard's diadynamics is one of the possible resources, but with little evidence in the literature. Objective: To analyze the effects of Bernard's diadinamics on nociception and edema in rats' tendon trauma. Materials and methods: The sample consisted of 18 Wistar rats divided into: control group (CG); negative pole Group (GP-); and positive pole Group (GP+). The animals had their right calcaneous tendon, injured with energy of 0.4 J. To assess nociception, we used the "von Frey" type digital analgesimeter, which was applied in the injured tendon, and to evaluate the edema, we used a caliper. The treatment was carried out with the current DF, CP and LP (3 minutes each), daily, for five therapies. Results: For the assessment of nociception, a significant difference with the time post-injury, only the last two assessments in GP+. In the evaluation of edema was obtained returned to baseline values only for GP+. Conclusion: Only Bernard's diadinamics positive pole was effective in reducing nociception and edema in rats subjected to the calcaneous tendon trauma.

Keywords: Achilles tendon. Transcutaneous electric nerve stimulation. Pain measurement.

\section{Introdução}

O tendão calcâneo apresenta grande importância, para o perfeito funcionamento da mecânica do tornozelo, desenvolvimento da marcha, corrida, salto e dança, com precisão (1). É o maior e mais resistente tendão do corpo humano, sendo um tipo de tecido conjuntivo denso, formado pela porção distal dos músculos gastrocnêmios e sóleo, o qual tem a função de transmitir a força desses músculos à porção posterior do calcâneo, determinando a flexão plantar do tornozelo $(2,3)$.

Há vários tipos de lesões que podem acometer o músculo esquelético e seus tendões. Essas lesões podem ter origem traumática ou ser resultado de processos lesivos, provocados por sofrimento prolongado, exercício intenso e forças de tração ou distração (1). Assim, quando os tendões são lesados, o corpo inicia um processo de cura, que pode ser dividido em fases que se sobrepõem: inflamação, proliferação e remodelamento $(4,5)$.

0 edema e a dor são alguns dos sinais que ocorrem durante a fase inflamatória. Na situação aguda decorrente de trauma, a estimulação direta das terminações nervosas livres da dor por substâncias químicas liberadas dentro dos tecidos danificados são responsáveis por ativar as vias de dor. Uma vez que o caminho da dor foi ativado, os impulsos chegam ao sistema nervoso central, onde a integração e interpretação ocorrem (6).

A formação do edema pode ocorrer praticamente em qualquer trauma, incluindo lesões atléticas. No entanto, o edema descontrolado pode aumentar a dor, prolongar a imobilização, reduzir a amplitude de movimento articular, e inibir a cicatrização, com isso pode se estender o tempo de recuperação do tecido (7).

A fisioterapia utiliza-se de técnicas ou combinação de tratamentos para o atendimento da dor e do edema pós-traumático, que incluem gelo, compressão, elevação, contrações do músculo esquelético e estimulação elétrica (8). Com relação à eletroestimulação, as Correntes diadinâmicas de Bernard (CDB), desenvolvidas na França em meados de 1950 por P. Bernard (9), são correntes polarizadas, de impulsos semissenoidais e de baixa frequência, existindo seis variedades dessas correntes, com variações de acordo com a frequência e modulação: monofásica fixa, difásica fixa, curtos períodos, longos períodos, rítmo sincopado e monofásica modulada (10).

Essas correntes produzem uma ação estimulante sobre os nervos sensitivos, causando vasodilatação e aumento do fluxo sanguíneo. Além disso, aumentam o metabolismo, o retorno venoso e linfático, previnem a formação de aderências, reduzem o inchaço e aliviam a dor (11). Contudo, apesar de 
usadas clinicamente, a literatura é pobre com respeito ao uso das correntes diadinâmicas em diversas afecções, principalmente com respeito ao seu uso em tendinopatias, justificando-se estudos para sua comprovação. Dessa maneira, o objetivo do presente estudo foi analisar os efeitos das CDB sobre a dor e edema no trauma tendíneo de ratos.

\section{Materiais e métodos}

O experimento foi realizado na Universidade Estadual do Oeste do Paraná (Unioeste) e foi aprovado pelo Comitê de Ética em Experimentação Animal e Aulas Práticas sob parecer n. 2210.

Foram utilizados 18 ratos Wistar machos, com idade média de 10 semanas, divididos aleatoriamente em três grupos: GC $(n=6)$ - os animais não receberam qualquer forma de corrente, servindo como controle na avaliação do processo evolutivo da dor e do edema; GP- ( $n=6)$ - os animais receberam aplicação do pólo negativo da corrente sobre o tendão do calcâneo; $\mathrm{GP}+(\mathrm{n}=6)$ - os animais receberam aplicação do pólo positivo da corrente sobre o tendão do calcâneo.

Modelo experimental de trauma no tendão do calcâneo

Os animais foram anestesiados com mistura de quetamina (95 mg/kg) e xilazina (12 mg/kg) e posicionados em decúbito lateral esquerdo, expondo a região lateral do tendão calcâneo direito ao trauma. Para a produção da lesão traumática foi utilizado um equipamento projetado pelo Departamento de Engenharia Civil da Unioeste, o qual consiste de um peso $(575 \mathrm{~g})$ partindo sempre de uma mesma altura inicial $(7 \mathrm{~cm})$ em queda sobre a face lateral do tendão calcâneo direito, totalizando uma energia de impacto de aproximadamente 0,40 J (12).

\section{Avaliação da nociceptividade}

Para a avaliação da nociceptividade, foi utilizado um teste com analgesímetro digital tipo filamento de "Von Frey" da marca Insight ${ }^{\circledR}$ (13), o qual foi aplicado no local em que houve o trauma tendíneo. Para realizar a compressão no local da lesão, o animal era contido manualmente e houve contato do filamento no tendão calcâneo, até que o animal retirasse seu membro.

As avaliações aconteceram da seguinte forma: primeira avaliação (AV1) com pré-lesão, seguida de procedimento traumático; a segunda avaliação (AV2) foi efetuada uma hora depois; a terceira avaliação (AV3) aconteceu ao final do primeiro tratamento; a quarta avaliação (AV4) ocorreu 24 horas após a lesão; no quinto dia da lesão ocorreu a AV5 logo após o último dia de tratamento; e, finalmente, no sexto dia procedeu-se à AV6, 24 horas após o último tratamento.

\section{Avaliação do edema no tendão do calcâneo}

Para analisar o edema no tendão calcâneo, foi calculado o diâmetro dos tendões em seu terço médio, com paquímetro posicionado médio-lateralmente. Essa forma de avaliação foi realizada nos mesmos períodos que a avaliação da dor (12).

\section{Protocolo de tratamento}

Logo depois de transcorrida a avaliação do momento pós-lesão, iniciou-se o tratamento dos tendões traumatizados, utilizando o aparelho de diadinâmicas, Dyadinaction standart da KW ${ }^{\circledR}$. Foram utilizadas as formas de corrente DF, CP e LP, por 3 minutos cada. A variação do polo ativo ocorreu de acordo com o grupo avaliado. 0 eletrodo ativo foi colocado em meio aquático e o membro da lesão também foi ali imerso; o eletrodo passivo foi posicionado na região lombar do animal, previamente submetida a tricotomia.

0 tratamento ocorreu nos grupos GP+ e GP-, no decorrer de cinco dias consecutivos, incluindo o dia da lesão, perfazendo um total de cinco terapias. No GC, os animais não receberam essas correntes, mas seu membro inferior direito, o qual teve o tendão lesionado, foi mantido em um recipiente com água. Após todos os tratamentos e avaliações, os animais foram submetidos a eutanásia por decapitação em guilhotina.

\section{Análise estatística}

Os resultados foram apresentados por média e desvio padrão e analisados por meio do teste de ANOVA 
medidas repetidas, para comparação dentro dos grupos, e ANOVA unidirecional, para comparações entre os grupos; em todos os casos foi utilizado o pós-teste Tukey e o nível de significância aceito foi $\alpha=0,05$.

\section{Resultados}

\section{Avaliação da nociceptividade}

Tanto para o GC como para o GP-, todos os momentos avaliados, na comparação com a AV1, apresentaram diferença significativa; sendo assim, houve aumento da nociceptividade e não retornou aos valores iniciais. Também o GP+ apresentou diferença significativa entre a AV1 e as demais avaliações. Contudo, no GP+ considerando-se os valores da AV2 com as avaliações seguintes, houve diferença na comparação com AV5 e AV6, com elevação no limiar nas duas últimas (Gráfico 1).

Nas comparações entre grupos, houve diferença significativa entre GC e GP+ em AV4 ( $p<0,05)$; também houve diferença significativa entre GC com GP- e $\mathrm{GP}+$ em AV5 $(\mathrm{p}<0,05) .0$ grupo controle apresentou menor limiar nociceptivo nessas comparações.

\section{Avaliação do edema}

Tanto para GC quanto para GP-, em todos os momentos avaliados, ao comparar com a AV1, houve aumento significativo dos diâmetros, ou seja, houve edema, o qual não retornou aos valores basais. Ao realizar as comparações entre os valores da AV2 com as demais avaliações, foi encontrada variação significativa apenas na quinta e na sexta avaliação, ou seja, o edema diminuiu nas duas últimas avaliações, para todos os grupos, inclusive o controle. Contudo, para GP+, ao comparar os valores basais (AV1) com AV5 e AV6 não houve diferença, ou seja, houve restauração dos valores, diferente do ocorrido para GC e GP- (Gráfico 2). Na comparação entre os grupos, não houve diferenças significativas.

\section{Discussão}

Nessa pesquisa, pretendeu-se analisar os efeitos das CDB sobre a dor e edema no trauma tendíneo de ratos. Dessa maneira, para o tratamento dos tendões lesionados, foram utilizados os polos positivo e negativo com as correntes DF, CP e LP, sendo que a amostra dividida em três grupos: GP-, GP+ e GC.

De acordo com os resultados obtidos, tanto o edema, que foi avaliado pelo paquímetro, como a nociceptividade, avaliada pelo analgesímetro digital, apresentaram resultados favoráveis de evolução, ou seja, houve diminuição no edema e aumento do limiar nociceptivo, quando realizado tratamento com o polo positivo. Entretanto, observa-se que não ocorreu o mesmo com o polo negativo das CDB.

Urrutia et al. (11) utilizaram as mesmas correntes utilizadas no presente estudo, com corrente anódica sobre a região articular, porém com durações

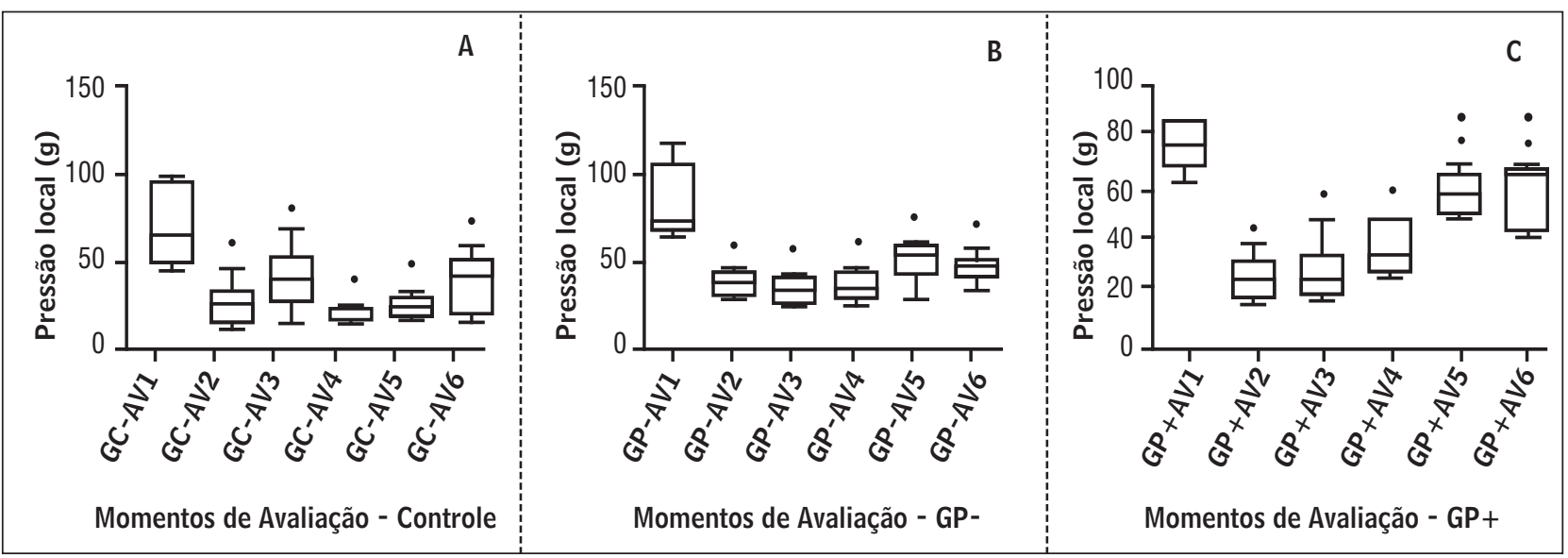

Gráfico 1 - Valores de limiar de retirada da pata (nociceptividade), em gramas, dos grupos controle (A), GP- (B) e GP+ (C), nos seis momentos de avaliação

Legenda: * = diferença significativa ao comparar com a AV1; $\bullet$ = diferença significativa ao comparar com AV2.

Fonte: Dados da pesquisa.

Fisioter Mov. 2013 jul/set;26(3): 617-22 


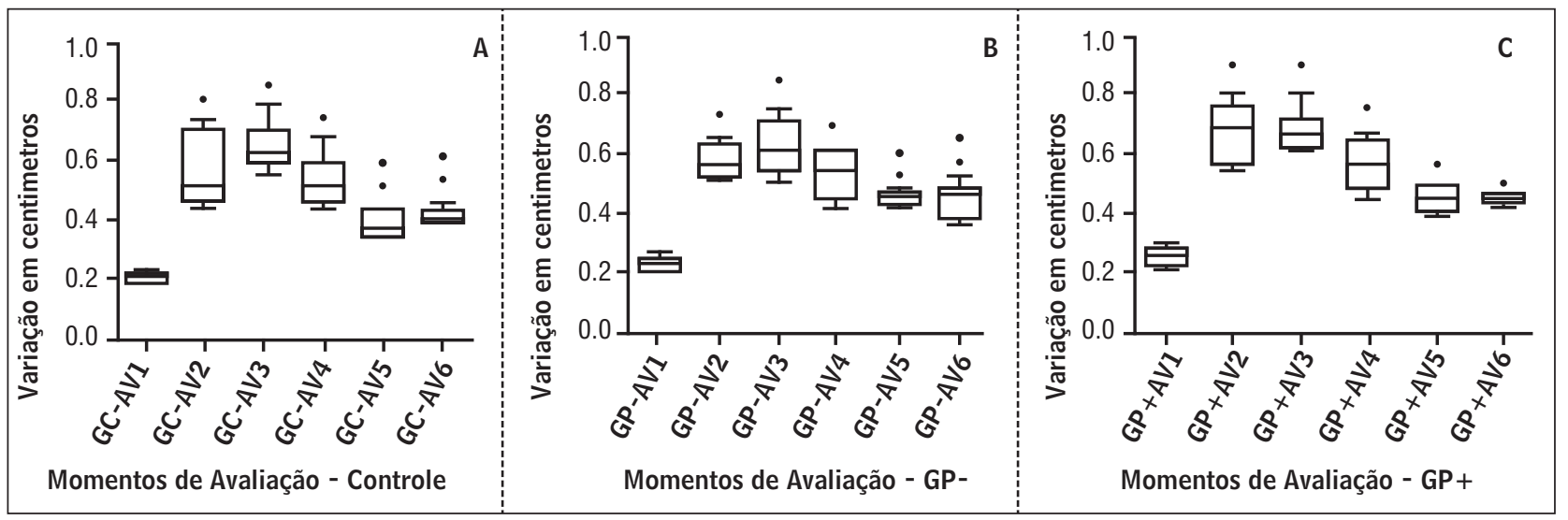

Gráfico 2 - Valores do diâmetro tendíneo (edema), em centímetros, dos grupos controle (A), GP- (B) e GP+ (C), nos seis momentos de avaliação

Legenda: * = diferença significativa ao comparar com a AV1; • = diferença significativa ao comparar com AV2.

Fonte: Dados da pesquisa.

diferentes (1 minuto de DF, 2 minutos de CP e 3 minutos de LP) e variando entre 10 a 12 sessões. Os autores observaram importante ação analgésica das diadinâmicas em pacientes com disfunção temporomandibular. Carvalho et al. (14), utilizando o eletrodo positivo sobre a região lombar e o negativo no trajeto do nervo isquiático de indivíduos com dor lombar crônica e ou lombociatalgia, perceberam efeitos analgésicos das correntes DF e LP (5 minutos cada) ao longo de sete terapias. Já Rossoni, Nakayama e Bertolini (15) observaram efeitos benéficos da corrente catódica (polo negativo como ativo) em indivíduos com DTM, tanto por escala visual analógica de dor, quanto pelo questionário de dor de McGill.

Uma das explicações na literatura para os efeitos positivos do polo positivo sobre a dor é a formação de ácido sob esse pólo, liberando oxigênio, o que pode ter um profundo efeito facilitador na recuperação de lesões. Além disso, é relatada uma ação de indução de bloqueio de impulsos dolorosos por excitação infralimiar permanente conduzindo a elevação do limiar da dor (9). Contudo, a última hipótese pode ser afastada no presente estudo, visto que tal fenômeno ocorreria nos dois polos e principalmente pelo fato que houve a necessidade de somação de terapias para a obtenção de resultados positivos sobre o limiar nociceptivo. Além disso, no pólo negativo das diadinâmicas ocorre um aumento importante do fluxo sanguíneo (9), o que poderia ter produzido resultados positivos em médio prazo, mas esse resultado não foi o encontrado.

Com respeito ao edema, notou-se que a evolução natural nesse tipo de lesão é sua significativa diminuição a partir do quinto dia, porém o único grupo que apresentou restauração dos valores foi o atendido pelo polo positivo. Guirro e Guirro (9) salientam que, segundo a lei de Cohen, quando a água é contida em rede de cavidades muito delgadas, aparecem cargas elétricas no sistema, sendo que a água apresenta características positivas, ou seja, seria repelida pelo polo positivo. Tal fato foi detectado por Reinert et al. (16), que, analisando o efeito imediato das diadinâmicas de Bernard na redução de edema em lesões traumáticas agudas em patas de ratas, verificaram que o polo positivo foi eficaz na redução do edema e o polo negativo produziu um ligeiro aumento, comparado ao controle. Contudo, acredita-se que os resultados positivos do polo positivo não ocorreram pela endosmose, pois os resultados logo após o tratamento e os observados após 24 horas não evidenciaram efeito benéfico da terapia.

Salienta-se que a ausência de análise histológica e bioquímica são limitações do presente estudo e podem ser abordadas em futuros estudos, contribuindo para ao uso dessa modalidade aplicada em lesões tendíneas.

\section{Conclusão}

O polo positivo das CDB foi eficaz em aumentar o limiar nociceptivo à pressão e reduzir o edema em ratos submetidos ao trauma no tendão de calcâneo. 0 polo negativo, ao contrário, não apresentou resultados favoráveis, obtendo comportamento levemente superior ao do grupo controle, pois apenas 
na comparação entre grupos mostrou maior limiar nociceptivo em AV4 na comparação com GC.

\section{Agradecimentos}

Ao Conselho Nacional de Desenvolvimento Científico e Tecnológico (CNPq), pelo apoio à pesquisa via concessão de bolsa de iniciação científica.

\section{Referências}

1. Nery CAS, Alloza JFM, Laurino CFS, Tanaka GS. Avaliação da força muscular isocinética do pé e tornozelo após tratamento cirúrgico das lesões do tendão de Aquiles, utilizando a transferência do tendão fibular curto. Rev Bras Ortop. 1997;32(7):503-12.

2. Arruda ERB, Rodrigues NC, Taciro C, Parizotto NA. Influência de diferentes comprimentos de onda da laserterapia de baixa intensidade na regeneração tendínea do rato após tenotomia. Rev Bras Fisioter. 2007;11(4):283-8.

3. Mayer A, Frasson VB, Ott R, Fortuna RO, Vaz MA. Desequilíbrios musculares entre flexores dorsais e plantares do tornozelo após tratamento conservador e acelerado da ruptura do tendão calcâneo. Fisioter Pesq. 2010;17(2):108-13.

4. Sandrey MA. Acute and chronic tendon injuries: factors affecting the healing response and treatment. J Sport Rehabil. 2003;12(1):70-91.

5. Lin TW, Cardenas L, Soslowsky LJ. Biomechanics of tendon injury and repair. J Biomech. 2004;37(6):865-77.

6. Domenico G. Pain relief with interferential therapy. Aust J Physiother. 1982;28(3):14-8.

7. Dolan MG, Mychaskiw AM, Mendel FC. Cool-water immersion and high-voltage electric stimulation curb edema formation in rats. J Athl Train. 2003;38(3):225-30.

8. Taylor K, Fish DR, Mendel FC, Burton HW. Effect of electrically induced muscle contractions on posttraumatic edema formation in frog hind limbs. Phys Ther. 1992;72(2):127-32.
9. Guirro E, Guirro R. Fisioterapia dermato-funcional. 3. ed. São Paulo: Manole; 2004.

10. León IG, Solana LS, García J. Corrientes diadinámicas y ultrasonido en el tratamiento de las disfunciones temporomandibulares. Rev Cubana Estomatol. 1998;35(3):80-5.

11. Urrutia ZEA, Solano LS, Rodríguez LL. Corrientes diadinámicas y galvánicas el tratamiento de la disfuncione emporomandibular. Rev Cubana Estomatol. 1998;35(3):73-9.

12. Bertolini GRF, Silva TS, Ciena AP, Trindade DL. Efeitos do laser de baixa potência sobre a dor e edema no trauma tendíneo de ratos. Rev Bras Med Esporte. 2008;14(4):362-6.

13. Vivancos GG, Verri WA Junior, Cunha TM, Schivo IRS, Parada CA, Cunha FQ, et al. An electronic pressure-meter nociception paw test for rats. Braz J Med Biol Res. 2004;37(3):391-9.

14. Carvalho AR, Fungueto EM, Canzi IM, Barbieiro C, Moraes V, Bertolini GRF, et al. Correntes diadinâmicas de Bernard e iontoforese no tratamento da dor lombar. Fisioter Mov. 2005;18(4):11-9.

15. Rossoni MA, Nakayama GK, Bertolini GRF. Correntes diadinâmicas de Bernard com e sem iontoforese na DTM: ensaio clínico randomizado. Arq Ciênc Saúde Unipar. 2009;13(1):3-8.

16. Reinert TC, Gonzalez TC, Siqueira FCHN, Loth EA, Bertolini GRF. Uso de correntes diadinâmicas de Bernard em edema agudo traumático em patas de ratas. Reabilitar. 2005;7(26):24-8.

Recebido: $18 / 08 / 2012$ Received: 08/18/2012

Aprovado: 26/04/2013

Approved: 04/26/2013 